\title{
A HOMOTOPY THEORY OF PRO-SPACES
}

\author{
BY \\ JERROLD W. GROSSMAN
}

\begin{abstract}
The category of towers of spaces, $\ldots \rightarrow X_{s+1} \rightarrow X_{s} \rightarrow$ $\ldots \rightarrow X_{0}$, viewed as pro-spaces, appears to be useful in the study of the relation between homology and homotopy of nonsimply connected spaces. We show that this category admits the structure of a closed model category, in the sense of Quillen; notions of fibration, cofibration, and weak equivalence are defined and shown to satisfy fundamental properties that the corresponding notions satisfy in the category of spaces. This enables one to develop a "homotopy theory" for pro-spaces.
\end{abstract}

1. Introduction. Recent work on the relation between homology and homotopy of nonsimply connected spaces (e.g. [2] , [3] , and unpublished results of E. Dror and W. Dwyer) involves towers of spaces, $\cdots \rightarrow X_{s+1} \rightarrow$ $X_{s} \rightarrow \cdots \rightarrow X_{0}$, viewed as pro-spaces. It would be helpful to be able to treat the category of such towers, which we denote by tow-S, like the category of spaces. In this paper we show that notions of fibration, cofibration, and weak equivalence can be defined for tow-S which give the category the structure of a closed model category, in the sense of Quillen [5]; thus one can "do homotopy theory" in tow-S.

In $\S 2$ we define the category tow-S, which is just a subcategory of the category of pro-spaces, and elucidate the maps in the category. Next ( $\S 3)$ we recall the definition of a closed model category, listing the axioms that fibrations, cofibrations, and weak equivalences must satisfy; topological spaces and simplicial sets are familiar examples of closed model categories. The definitions of fibration, cofibration, and weak equivalence for tow-S are given in $\S 4$, and the proof that tow-S is a closed model category with these definitions occupies $\S \S 5-8$.

We work over the category $S$ of simplicial sets [4], but the definitions and proofs are not combinatorial.

The author thanks Daniel M. Kan for his advice and suggestions in the

Presented to the Society, October 27, 1973; received by the editors November 14, 1973.

AMS (MOS) subject classifications (1970). Primary 55F65, 55D05, 55D10; Secondary $18 \mathrm{E} 35$.

Key words and phrases. Pro-space, pro-map, tower of spaces, model category, weak equivalence, fibration, cofibration. 
research and writing of this paper, and William Dwyer for many helpful conversations.

2. Pro-spaces. In this section we introduce the category tow-S.

2.1. Definition. The category tow-S is the category whose objects are towers in $S$ (the category of simplicial sets),

$$
\cdots \rightarrow X_{s+1} \rightarrow X_{s} \rightarrow \cdots \rightarrow X_{0},
$$

written $\left\{X_{s}\right\}$, and whose maps are given by

$$
\text { Hom }_{\text {tow }-s}\left(\left\{X_{s}\right\},\left\{Y_{s}\right\}\right)=\varliminf_{j}^{\lim } \underset{i}{\lim } \operatorname{Hom}_{s}\left(X_{i}, Y_{j}\right) \text {. }
$$

The reader familiar with Artin-Mazur [1] will recognize that tow-S is the full subcategory of pro-S with the index restricted to the nonnegative integers.

A map $\left\{X_{s}\right\} \rightarrow\left\{Y_{s}\right\}$ in tow-S is best thought of as a compatible system of maps $\left\{X_{t} \rightarrow Y_{s}\right\}_{s}$, modulo the relation that $X_{t} \rightarrow Y_{s}$ and $X_{u} \rightarrow Y_{s}$ are equivalent if for some large $w$ the maps $X_{w} \rightarrow X_{t} \rightarrow Y_{s}$ and $X_{w} \rightarrow X_{u} \rightarrow Y_{s}$ are the same. In particular any level map, i.e. tower of maps

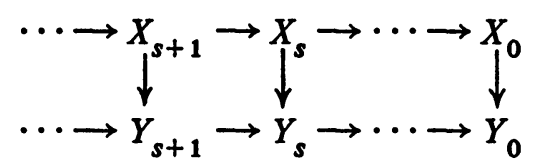

in $S$, represents a map $\left\{X_{s}\right\} \rightarrow\left\{Y_{s}\right\}$ in tow-S. Not every map $\left\{X_{s}\right\} \rightarrow$ $\left\{Y_{s}\right\}$ can be so presented, however. Instead we have

2.2. Definition. Let $\psi:\left\{X_{s}\right\} \rightarrow\left\{Y_{s}\right\}$ be a map in tow-S. We say that the level map $\left\{f_{s}: X_{s}^{\prime} \rightarrow Y_{s}^{\prime}\right\}$ is a level representative of $\psi$ if there are equivalences in tow-S, $\left\{X_{s}\right\} \approx\left\{X_{s}^{\prime}\right\}$ and $\left\{Y_{s}\right\} \approx\left\{Y_{s}^{\prime}\right\}$ such that the following diagram in tow-S commutes:

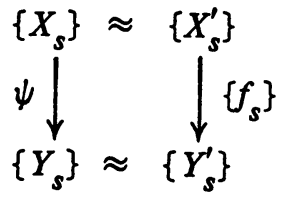

Thus for example the identity level map $\left\{\right.$ id: $X_{s} \rightarrow X_{s}$ \} is a level representative of any equivalence $\left\{X_{s}\right\} \approx\left\{Y_{s}\right\}$. Clearly any map in tow-S has a level representative, obtained simply by taking a subtower of the domain. We shall see in $\$ 4$, however, that the full generality of Definition 2.2 is useful.

3. Model categories. In this section we recall Quillen's definition of closed model category and observe that simplicial sets and topological spaces form closed model categories.

3.1. Definition [6]. A closed model category is a category $C$ to- 
gether with three classes of maps in $\mathcal{C}$, called fibrations, cofibrations, and weak equivalences, satisfying the following axioms:

CMI. $C$ is closed under finite direct and inverse limits.

CM2. If $f$ and $g$ are maps in $C$ such that $g f$ is defined, and two of $f, g$, and $g f$ are weak equivalences, so is the third.

CM3. If a map $f$ is a retract of a map $g$ (i.e. if the diagram

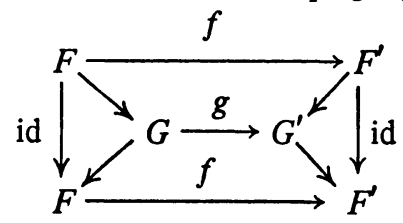

commutes), and $g$ is a fibration, cofibration, or weak equivalence, then so is $f$.

CM4. Given the following solid arrow diagram in $C$

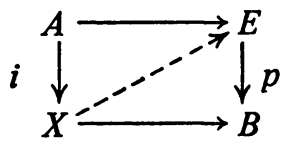

in which $i$ is a cofibration and $p$ is a fibration, the dotted arrow exists if either

(i) $p$ is a weak equivalence, or

(ii) $i$ is a weak equivalence.

CM5. Any map in $C$ can be factored in two ways:

(i) as a cofibration followed by a fibration which is also a weak equivalence;

(ii) as a cofibration which is also a weak equivalence followed by a fibration.

If the dotted arrow exists in the diagram of CM4, we say that $i$ has the left lifting property with respect to $p$, and $p$ has the right lifting property with respect to $i$. A map which is both a fibration [resp. cofibration] and a weak equivalence is called a trivial fibration [resp. trivial cofibration].

3.2. Examples. (See [5], [6, p. 259], and [2, Chapter VIII].) We use the fact that the category $S$ of simplicial sets is a closed model category, where fibrations are Kan fibrations (see $[4$, p. 25]), cofibrations are injective maps, and weak equivalences are weak homotopy equivalences, i.e. maps $X$ $\rightarrow Y$ such that $\pi_{0} X \rightarrow \pi_{0} Y$ is an isomorphism of sets and $\pi_{n}(X, *) \rightarrow$ $\pi_{n}(Y, *)$ is an isomorphism of groups for $n \geqslant 1$ and every choice of basepoint in $X$. (Here and throughout this paper, we denote the image of a basepoint * also by *.) Similarly the category $T$ of topological spaces is a closed model category, with Serre fibrations as fibrations, weak homotopy equivalences as weak equivalences, and retracts of sequences of relative $\mathrm{CW}$ complexes [7, 
p. 401] as cofibrations. In both of these examples, the associated homotopy category (obtained by localizing at, i.e. formally inverting, weak equivalences) is just the familiar homotopy category of Kan complexes, or, equivalently, of $\mathrm{CW}$ complexes.

4. Statement of results. Our main result is that fibrations, cofibrations, and weak equivalences can be defined in tow-S to satisfy the axioms CM1CM5 for a closed model category (3.1).

We begin with cofibration, which has the most natural definition.

4.1. Definition. A map in tow-S is a cofibration if it has a level representative $\left\{f_{s}: X_{s} \rightarrow Y_{s}\right\}$ such that each $f_{s}$ is a cofibration in $S$, i.e. an injective map of simplicial sets.

4.2. REMARK. The need for the generality in the definition of level representative (2.2) can be seen from the following example. Consider the tower $\left\{I_{s}\right\}$ which has a 1-simplex at each level and $I_{s+1}$ projected to the left endpoint of $I_{s}$. The map from $\left\{I_{s}\right\}$ to $\{*\}$, a tower of single points, is clearly an equivalence in tow-S, and hence must be $[6$, p. 234] a cofibration. But no level representative of $\left\{I_{s}\right\} \rightarrow\{*\}$ using subtowers of the domain and range is a tower of cofibrations.

The definition of weak equivalence is complicated by the fact that the spaces $X_{s}$ in an object $\left\{X_{s}\right\}$ of tow-S need not be connected or even nonempty.

4.3. Definition. A map in tow-S is a weak equivalence if it has a level representative $\left\{f_{s}: X_{s} \rightarrow Y_{s}\right\}$ such that

(i) $\left\{\pi_{0} f_{s}\right\}$ is an equivalence of pro-sets, i.e. for each $s \geqslant 0$ there is a $t \geqslant s$ and a map $\pi_{0} Y_{t} \rightarrow \pi_{0} X_{s}$ making the following diagram (of sets) commute:

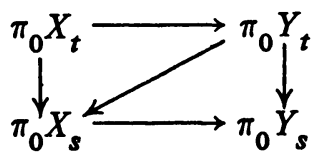

(ii) for each $n \geqslant 1$ and each $s \geqslant 0$ there is a $t \geqslant s$ such that for each choice of basepoint in $X_{t}$ there is a map $\pi_{n}\left(Y_{t}, *\right) \rightarrow \pi_{n}\left(X_{s}, *\right)$ making the following diagram (of groups) commute:

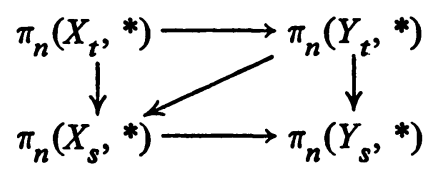

It is routine to check that if one level representative of a map satisfies conditions (i) and (ii), so does any other. In the case that all the spaces $X_{s}$ and $Y_{s}$ are connected and $\left\{X_{s}\right\}$ has a compatible basepoint, the definition 
becomes simply: a map $\left\{X_{s}\right\} \rightarrow\left\{Y_{s}\right\}$ is a weak equivalence if the induced maps $\left\{\pi_{n} X_{s}\right\} \rightarrow\left\{\pi_{n} Y_{s}\right\}$ are pro-isomorphisms for $n \geqslant 0$. This is the usual definition ([1], [2]).

Before defining fibration, it is convenient to introduce the following (partly standard [7, p. 404]) terminology for maps of simplicial sets.

4.4. Definition. Let $N$ be a nonnegative integer and let $f: X \rightarrow Y$ be a map in $S$. Then $f$ is an $N$-equivalence (for $X$ not empty) if for every choice of basepoint in $X, \pi_{n}(X, *) \rightarrow \pi_{n}(Y, *)$ is an isomorphism for $0 \leqslant n<N$ and an epimorphism for $n=N$. (If $X$ is empty, then $X \rightarrow Y$ is an $N$-equivalence if $Y$ is also empty.) On the other hand, $f$ is a co-N-equivalence if for every choice of basepoint in $X, \pi_{n}(X, *) \rightarrow \pi_{n}(Y, *)$ is an isomorphism for $n>N$ and a monomorphism for $n=N$.

We define fibrations in two stages.

4.5. Definition. A map in tow-S is a level-fibration if it has a level representative $\left\{X_{s} \rightarrow Y_{s}\right\}$ such that for each $s$

(i) there exists an integer $N(s)$ such that $X_{s} \rightarrow Y_{s}$ is a co- $N(s)$-equivalence;

(ii) $X_{s} \rightarrow Y_{s}$ is a fibration in $S$; and

(iii) the induced map $X_{s+1} \rightarrow X_{s} \times_{Y_{s}} Y_{s+1}$ is a fibration in $S$.

Induction and diagram chasing show that condition (iii) implies that for each $k \geqslant 1$ the induced map $X_{s+k} \rightarrow X_{s} \times_{Y_{s}} Y_{s+k}$ is a fibration. In particular a subtower of a level representative satisfying Definition 4.5 also satisfies Definition 4.5.

4.6. Definition. A map in tow-S is a fibration if it is a retract (3.1, Axiom CM3) of a level-fibration (4.5).

In particular a level-fibration is a fibration.

4.7. ThEOREM . The category tow-S, with fibrations, cofibrations, and weak equivalences defined above, is a closed model category.

Organization of Proof. Axiom CM1 is proved by Artin-Mazur [1, Proposition A.4.2]. In $\S 5$ we prove CM2 and CM3. In $\S 6$ we reduce CM4 and CM5 to more concrete lifting and factoring statements, which are then proved in $\S 8$ and $\S 7$, respectively.

4.8. REMARK. Everything can just as easily be done for tow- $S_{*}$, where $S_{*}$ is the category of pointed, but not necessarily connected, simplicial sets. The fixed basepoint would play no role in the definitions or proofs, except to exclude the case of empty simplicial sets.

\section{Proofs of CM2 and CM3.}

PROOF OF CM2. The proof consists of setting up the right level diagram. 
Suppose $\left\{X_{s}\right\} \rightarrow\left\{Y_{s}\right\} \rightarrow\left\{Z_{s}\right\}$ are maps in tow-S. It is easy to see that by taking level representatives (2.2) we can assume that we have a tower $\left\{X_{s} \rightarrow\right.$ $\left.Y_{s} \rightarrow Z_{s}\right\}$. We shall consider the case in which $\left\{X_{s}\right\} \rightarrow\left\{Z_{s}\right\}$ and $\left\{X_{s}\right\} \rightarrow$ $\left\{Y_{s}\right\}$ are weak equivalences; the other two cases are similar but easier. We want to show that $\left\{Y_{s}\right\} \rightarrow\left\{Z_{s}\right\}$ is a weak equivalence, i.e. (4.3) for each $n \geqslant 1$ and $s \geqslant 0$ there is a $t \geqslant s$ such that for each choice of basepoint $\cdot$ in $Y_{t}$ there is a map $\pi_{n}\left(Z_{t}, \cdot\right) \rightarrow \pi_{n}\left(Y_{s}, \cdot\right)$ making the following diagram commute

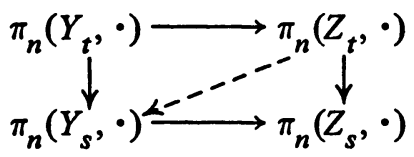

and a similar statement for $\pi_{0}$. The case of $\pi_{0}$ is easy, so fix $n \geqslant 1, s \geqslant 0$. Since $\left\{X_{s}\right\} \rightarrow\left\{Z_{s}\right\}$ is a weak equivalence, we can choose $w \geqslant s$ so that the dotted arrow exists in the following diagram for every choice of basepoint in $X_{w}$ :

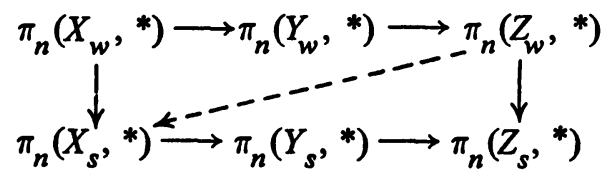

Next, since $\left\{X_{s}\right\} \rightarrow\left\{Y_{s}\right\}$ is a weak equivalence, we can choose $u \geqslant w$ so that the dotted arrow exists in the following diagram for every choice of basepoint in $X_{u}$ :

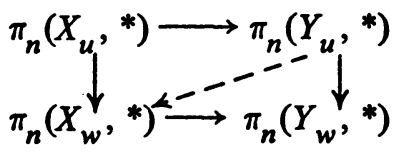

Finally we choose $t \geqslant u$ so that the dotted arrow exists in the following diagram:

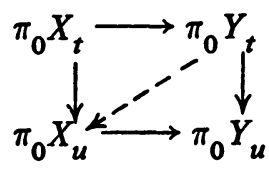

Now let - be any basepoint in $Y_{t}$. Because of the last diagram, the image of $\cdot$ in $Y_{u}$ is in a (path) component which is in the image of $X_{u} \rightarrow Y_{u}$. Choose a basepoint + in $X_{u}$ such that (the images of) + and - are in the same (path) component in $Y_{u}$, and let $\alpha$ be a path between them. Then $\alpha$ induces an isomorphism

$$
\alpha_{\#}: \pi_{n}(A,+) \longrightarrow \pi_{n}(A, \cdot)
$$

for $A=Y_{u}, Z_{u}, Y_{w}, Z_{w}, Y_{s}, Z_{s}$; and $\alpha_{\#}$ and $\alpha_{\#}^{-1}$ commute with the appropriate maps. Define the desired map as the composite 


$$
\begin{aligned}
\pi_{n}\left(Z_{t}, \cdot\right) & \longrightarrow \pi_{n}\left(Z_{u}, \cdot\right) \stackrel{\alpha_{\#}^{-1}}{\longrightarrow} \pi_{n}\left(Z_{u},+\right) \\
& \longrightarrow \pi_{n}\left(Z_{w},+\right) \longrightarrow \pi_{n}\left(X_{s},+\right) \rightarrow \pi_{n}\left(Y_{s},+\right) \stackrel{\alpha_{\#}}{\longrightarrow} \pi_{n}\left(Y_{s}, \cdot\right) .
\end{aligned}
$$

A diagram chase gives the desired commutativity relations.

PROOF OF CM3 FOR WEAK EQUIVALENCE. We are given the following commutative diagram in tow-S:

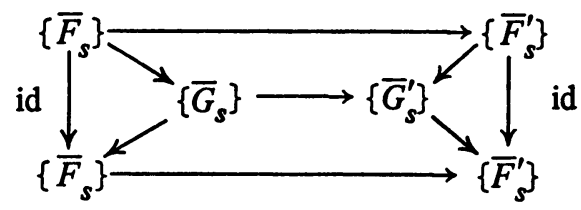

Taking appropriate subtowers we get the following "level" representative of the diagram:

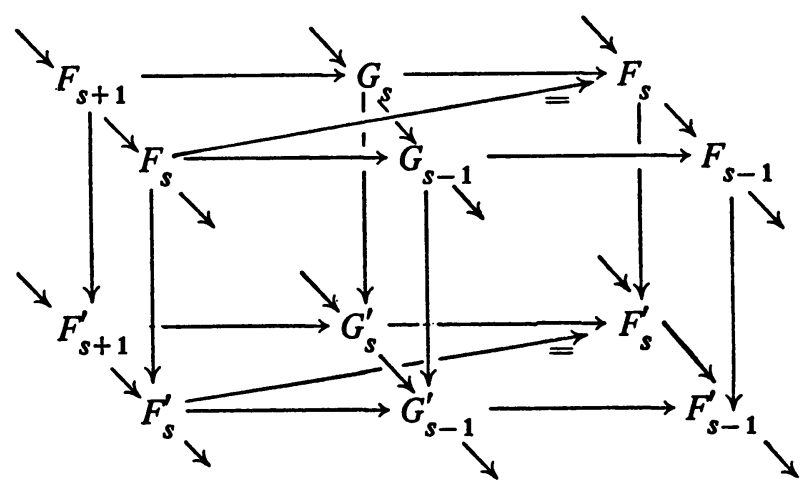

By diagram chasing one can verify that the definition of weak equivalence is satisfied by $\left\{F_{s} \rightarrow F_{s}^{\prime}\right\}$ if it is satisfied by $\left\{G_{s} \rightarrow G_{s}^{\prime}\right\}$.

PROOF OFCM3 For COFIBRATION. It is clear from the above diagram that the following tower

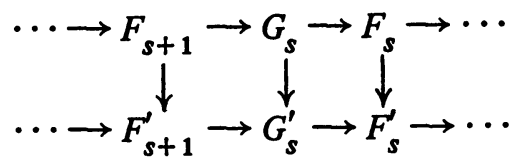

is a. level representative for $\left\{\bar{F}_{s}\right\} \rightarrow\left\{\bar{F}_{s}^{\prime}\right\}$. Hence so is the subtower consisting only of the $G_{s} \rightarrow G_{s}^{\prime}$. Now if $\left\{\bar{G}_{s}\right\} \rightarrow\left\{\bar{G}_{s}^{\prime}\right\}$ is a cofibration, we could have chosen the level representative in such a way (4.1) that each $G_{s} \rightarrow G_{s}^{\prime}$ is a cofibration in $S$; hence $\left\{\bar{F}_{s}\right\} \rightarrow\left\{\bar{F}_{s}^{\prime}\right\}$ is also a cofibration.

The proof of CM3 for fibration is immediate from Definitions 4.5 and 4.6.

6. Proofs of CM4 and CM5. In this section we reduce CM5 and CM4 to more concrete factoring and lifting axioms, which are proved in $\S \S 7-8$. 
First we show that it suffices to prove the factoring and lifting axioms for level-fibrations (4.5), namely

Any map in tow-S can be factored as a cofibration followed

$F(i) \quad$ by a trivial level-fibration (i.e. a level-fibration which is also a weak equivalence).

$F(i i) \quad$ Any map in tow-S can be factored as a trivial cofibration followed by a level-fibration.

$L(i) \quad$ Cofibrations have the left lifting property with respect to trivial level-fibrations.

Trivial cofibrations have the left lifting property with respect to level-fibrations.

Indeed, CM5 follows from $F(\mathrm{i})$ and $F($ ii), while CM4(ii) is a straightforward application of Definition 4.6 and $L$ (ii). Then to prove CM4(i), we take the given lifting problem in tow-S

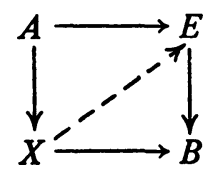

(using Roman letters for objects of tow-S in this proof only), in which $A \rightarrow X$ is a cofibration and $E \rightarrow B$ is a trivial fibration, and factor $E \rightarrow B$ by $F(\mathrm{i})$ into $E \rightarrow Y \rightarrow B$, where $Y \rightarrow B$ is a trivial level-fibration and $E \rightarrow Y$ is a cofibration, which is trivial by CM2. Then in the following diagram

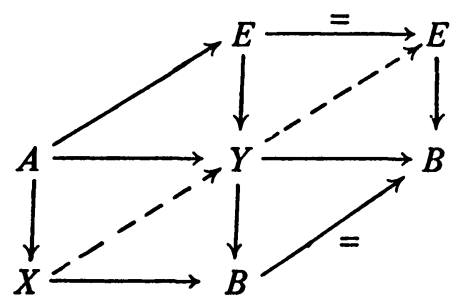

the dotted arrow $X \rightarrow Y$ exists by $L(\mathrm{i})$, and the dotted arrow $Y \rightarrow E$ exists by CM4(ii). Their composite is the desired lifting $X \rightarrow E$.

Next, to make the proofs easier, we will modify $F($ ii) and $L$ (ii) slightly.

6.1. Definition. A map in tow-S is a level-trivial cofibration if it has a level representative $\left\{X_{s} \rightarrow Y_{s}\right\}$ such that for each $s, X_{s} \rightarrow Y_{s}$ is a cofibration and an s-equivalence.

Clearly a level-trivial cofibration is a trivial cofibration

We now replace $F($ ii) and $L$ (ii) by the following statements

$F\left(\right.$ ii) ${ }^{\prime}$

Any map in tow-S can be factored as a level-trivial cofibration followed by a level-fibration 
and observe that it suffices to prove $F(\mathrm{i}), F(\mathrm{ii})^{\prime}, L(\mathrm{i})$, and $L(\mathrm{ii})^{\prime}$. For it is clear that $F(\text { ii) })^{\prime}$ implies $F\left(\right.$ ii); and $L\left(\right.$ ii) follows from $L\left(\right.$ ii) ${ }^{\prime}, F(\text { (ii) })^{\prime}, L(\mathrm{i})$, and CM2 (the proof is similar to the proof of CM4(i), above). The factoring properties $F(\mathrm{i})$ and $F\left(\right.$ (ii) $^{\prime}$ are proved in $\S 7$, while the lifting properties $L(\mathrm{i})$ and $L(\mathrm{ii})^{\prime}$ are proved in $\S 8$.

7. Proof of factoring properties $F(\mathrm{i})$ and $F(\mathrm{ii})^{\prime}$. In this section we prove that any map in tow-S, $\left\{X_{s}\right\} \rightarrow\left\{Y_{s}\right\}$, can be factored

$F\left(\right.$ ii) ${ }^{\prime}$ as $\left\{X_{s}\right\} \rightarrow\left\{Z_{s}\right\} \rightarrow\left\{Y_{s}\right\}$ with $\left\{X_{s}\right\} \rightarrow\left\{Z_{s}\right\}$ a cofibration and $\left\{Z_{s}\right\} \rightarrow\left\{Y_{s}\right\}$ a trivial level-fibration (4.5); and as $\left\{X_{s}\right\} \rightarrow\left\{Z_{s}\right\} \rightarrow\left\{Y_{s}\right\}$ with $\left\{X_{s}\right\} \rightarrow\left\{Z_{s}\right\}$ a level-trivial cofibration (6.1) and $\left\{Z_{s}\right\} \rightarrow\left\{Y_{s}\right\}$ a level-fibration.

The proof consists of factoring in an appropriate way each level of a level representative of $\left\{X_{s}\right\} \rightarrow\left\{Y_{s}\right\}$ and then modifying the result to fit Definition 4.5.

We shall use the following generalizations of the factoring and lifting properties for simplicial sets.

7.1. LEMMA. Given an integer $N \geqslant 0$ and a map $X \rightarrow Y$ in $S$, then there is a factoring $X \rightarrow Z \rightarrow Y$ such that $X \rightarrow Z$ is a cofibration and $N$-equivalence, and $Z \rightarrow Y$ is a fibration and co-N-equivalence (4.4).

7.2. LEMMA. Given an integer $N \geqslant 0$ and a solid arrow diagram in $S$

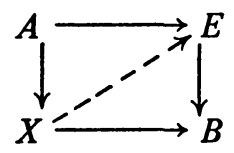

where $A \rightarrow X$ is a cofibration and Nequivalence, and $E \rightarrow B$ is a fibration and co-N-equivalence (4.4), then the dotted arrow exists.

We now prove $F(\mathrm{ii})^{\prime}$; the proof of $F(\mathrm{i})$ is similar, using the ordinary factoring and lifting axioms for $S$ instead of Lemmas 7.1 and 7.2. Let $\left\{X_{s}\right\}$ $\rightarrow\left\{Y_{s}\right\}$ be the given map in tow-S, which we can assume (2.2) has a level representative $\left\{X_{s} \rightarrow Y_{s}\right\}$. By Lemma 7.1 factor each level into $X_{s} \rightarrow A_{s} \rightarrow$ $Y_{s}$, with $X_{s} \rightarrow A_{s}$ a cofibration and s-equivalence, and $A_{s} \rightarrow Y_{s}$ a fibration and co-s-equivalence. By Lemma 7.2 the arrow $A_{s+1} \rightarrow A_{s}$ exists in the following diagram:

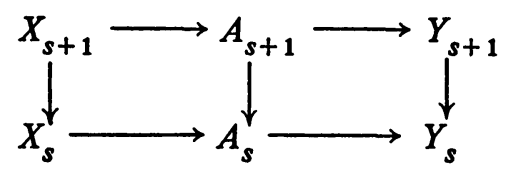

The problem now is that $\left\{A_{s}\right\} \rightarrow\left\{Y_{s}\right\}$ need not be a level-fibration because $A_{s+1} \rightarrow A_{s} \times_{Y_{s}} Y_{s+1}$ is not in general a fibration, so we must modify the middle terms. Let $Z_{0}=A_{0}$, and assume by induction that $Z_{0}, \cdots, Z_{s-1}$ 
have been defined to replace $A_{0}, \cdots, A_{s-1}$, so that the pull-back condition is satisfied through level $s-2$. Let $B_{s}=Z_{s-1} \times{ }_{Y_{s-1}} Y_{s}$ and by Lemma 7.1 factor $A_{s} \rightarrow B_{s}$ into $A_{s} \rightarrow Z_{s} \rightarrow B_{s}$ where $A_{s} \rightarrow Z_{s}$ is an sequivalence and cofibration, and $Z_{s} \rightarrow B_{s}$ is a co-s-equivalence and fibration:

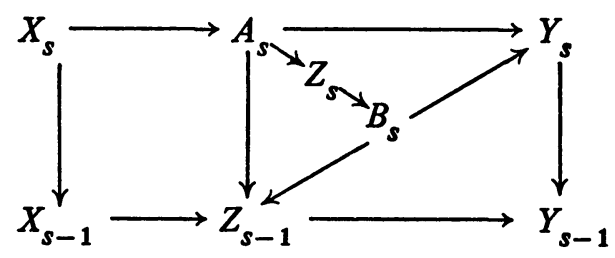

Then $\left\{Z_{s} \rightarrow Y_{s}\right\}$ is a level map satisfying Definition 4.5 , and $\left\{X_{s} \rightarrow Z_{s}\right\}$ is a level map satisfying Definition 6.1, as desired.

Proof OF 7.1. Essentially we just apply the well-known Moore-Postnikov factorization of a map. In detail, factor $X \rightarrow Y$ into $X \rightarrow A \rightarrow Y$, with $X \rightarrow A$ a trivial cofibration and $A \rightarrow Y$ a fibration. Then factor $A \rightarrow Y$ into $A \rightarrow B \rightarrow$ $Y$ where $B$ is the $(N-1)$ st Moore-Postnikov "part" of the fibration $A \rightarrow Y$ (see [4, p. 34], which contains the unneeded hypothesis that $A$ and $Y$ are connected Kan complexes). Thus $A \rightarrow B$ is a (fibration and) $N$-equivalence, and $B \rightarrow Y$ is a fibration and co- $N$-equivalence. Finally, factor $A \rightarrow B$ into $A \rightarrow Z \rightarrow B$ with $A \rightarrow Z$ a cofibration and $Z \rightarrow B$ a trivial fibration. Clearly $X \rightarrow Z$ $\rightarrow Y$ has the desired properties.

Proof OF 7.2. Because $A \rightarrow X$ is an $N$-equivalence, there is no obstruction to lifting the $N$-skeleton, $A \cup X^{N}$. Because $E \rightarrow B$ is a co- $N$-equivalence, there is then no obstruction to lifting the rest of $X$.

8. Proof of lifting properties $L(\mathrm{i})$ and $L(\mathrm{ii})^{\prime}$. In this section we prove that cofibrations have the left lifting property with respect to trivial level-fibrations (4.5),

and

$L$ (ii) $^{\prime} \quad$ level-trivial cofibrations (6.1) have the left lifting
property with respect to level-fibrations.

The proofs are similar, although $L(i)$ is more technical. In both cases the plan is to construct the lifting level by level. Given a level diagram representing the lifting problem

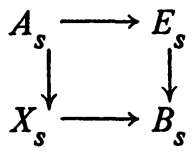


we want to find a monotonic function $p$ and maps $X_{p(s)} \rightarrow E_{s}$ such that $\left\{X_{p(s)} \rightarrow E_{s}\right\}$ defines a level map from a subtower of $\left\{X_{s}\right\}$ to $\left\{E_{s}\right\}$, i.e. the following diagram commutes

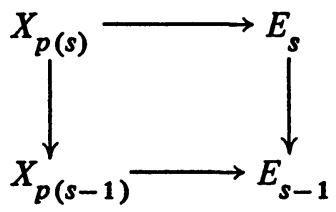

and the map is a lifting, i.e. the following diagram commutes:

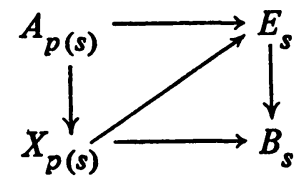

We first do the easier $L$ (ii)'. Given the following diagram in tow-S

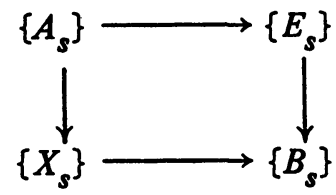

with $\left\{A_{s}\right\} \rightarrow\left\{X_{s}\right\}$ a level-trivial cofibration and $\left\{E_{s}\right\} \rightarrow\left\{B_{s}\right\}$ a level-fibration, it is not hard to see that we can assume that we have a level diagram

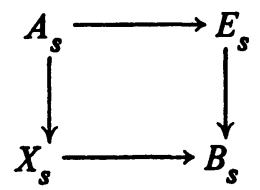

such that for each $s, A_{s} \rightarrow X_{s}$ is a cofibration and s-equivalence (6.1), and $E_{s} \rightarrow B_{s}$ is a fibration and co- $N(s)$-equivalence (4.5) for some integer $N(s)$.

To construct the lifting to $E_{0}$, let $p(0)=N(0)$. Then by Lemma 7.2 the dotted arrow exists in the following diagram:

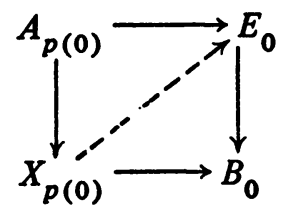

For the inductive step, assume that the map $X_{p(s-1)} \rightarrow E_{s-1}$ has been defined. Let $p(s)=\max (p(s-1)+1, N(s-1), N(s))$. Define $E_{s}^{\prime}=B_{s} \times_{B_{s-1}}$ $E_{s-1}$; from Definition 4.5 we know that $E_{s} \rightarrow E_{s}^{\prime}$ is a fibration. By universality the map $X_{p(s)} \rightarrow E_{s}^{\prime}$ exists in the following diagram: 


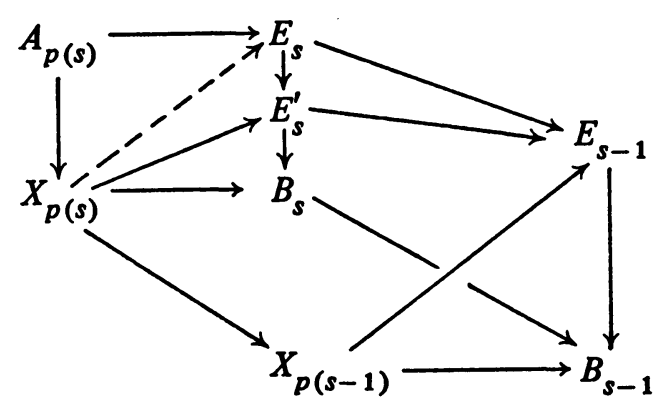

Now since the fibration $E_{s-1} \rightarrow B_{s-1}$ is a co-N(s-1)-equivalence, so is $E_{s}^{\prime} \rightarrow B_{s}$; and hence, since $E_{s} \rightarrow B_{s}$ is a co-N/s)-equivalence, $E_{s} \rightarrow E_{s}^{\prime}$ is a co-p $(s)$-equivalence. Thus the dotted arrow in the above diagram exists by Lemma 7.2. Note that it was necessary to lift $X_{p(s)}$ first to $E_{s}^{\prime}$ in order that $X_{p(s)} \rightarrow E_{s}$ be compatible with $X_{p(s-1)} \rightarrow E_{s-1}$.

For $L(\mathrm{i})$ we must construct the maps $X_{p(s)} \rightarrow E_{s}$ by skeleta $X_{p(s)}^{k}$, i.e. we will find compatible maps $A_{p(s)} \cup X_{p(s)}^{0} \rightarrow E_{s+m}, A_{p(s)} \cup X_{p(s)}^{1} \rightarrow$ $E_{s+m-1}, \cdots, A_{p(s)} \cup X_{p(s)}^{m} \rightarrow E_{s}$ and finally extend to $X_{p(s)} \rightarrow E_{s}$. To insure, moreover, that $X_{p(s)} \rightarrow E_{s}$ is compatible with $X_{p(s-1)} \rightarrow E_{s-1}$ we must, for each skeleton, first lift to an appropriate pull-back as in the proof of $L$ (ii)', above.

We will need two lemmas. The first establishes sufficient conditions for lifting skeleta, while the second shows how to choose a nice enough level representative of a trivial level-fibration.

8.1. Lemma. Let $N$ be a nonnegative integer, and consider the following solid arrow diagram in $S$, where $A \rightarrow X$ is a cofibration, and $E \rightarrow B$ and $E^{\prime} \rightarrow B^{\prime}$ are fibrations (we use $X^{r}$ to denote the $r$ skeleton of $X$, with $X^{-1}$ the empty space):

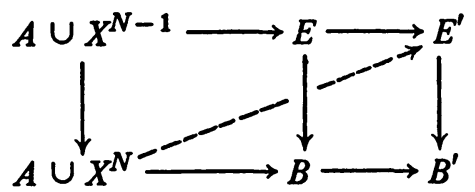

Then the dotted arrow exists if

(i) $N=0$ and the image of $\pi_{0} B$ in $\pi_{0} B^{\prime}$ is contained in the image of $\pi_{0} E^{\prime}$ in $\pi_{0} B^{\prime}$;

(ii) $N \geqslant 1$ and for each choice of basepoint in $E$, the map $\pi_{N-1}(F, *)$ $\rightarrow \pi_{N-1}\left(F^{\prime}, *\right)$ is the zero map, where $F$ and $F^{\prime}$ are the fibers of $E \rightarrow B$ and $E^{\prime} \rightarrow B^{\prime}$ over the basepoint.

PROOF. For $N=0$ the construction of the lifting is straightforward. For $N \geqslant 1$ condition (ii) implies that there is no obstruction to lifting to $E^{\prime}$. 
8.2. LEMMA. A trivial level-fibration in tow-S has a level representative $\left\{E_{s} \rightarrow B_{s}\right\}$ which satisfies Definition 4.5 and the following conditions:

(i) $\pi_{n}\left(F_{s+1}, *\right) \rightarrow \pi_{n}\left(F_{s}, *\right)$ is the zero map for each $n, s \geqslant 0$ and every choice of basepoint in $E_{s+1}$ (where $F_{i}$ is the fiber of $E_{i} \rightarrow B_{i}$ over the basepoint), and the image of $\pi_{0} B_{s+1}$ in $\pi_{0} B_{s}$ is contained in the image of $\pi_{0} E_{s}$ in $\pi_{0} B_{s}$.

(ii) Let $E_{s}^{\prime}=B_{s} \times{ }_{B_{r}} E_{r}$ and $E_{s+1}^{\prime}=B_{s+1} \times{ }_{B_{r+1}} E_{r+1}$, for $r \geqslant 0$, $s \geqslant r+2$. Choose a basepoint in $E_{s+1}$ and let $G_{i}$ be the fiber of $E_{i} \rightarrow E_{i}^{\prime}$ Then $\pi_{n}\left(G_{s+1},{ }^{*}\right) \rightarrow \pi_{n}\left(G_{s}, *\right)$ is the zero map for each $n \geqslant 0$, and the image of $\pi_{0} E_{s+1}^{\prime}$ in $\pi_{0} E_{s}^{\prime}$ is contained in the image of $\pi_{0} E_{s}$ in $\pi_{0} E_{s}^{\prime}$.

The proof is postponed until the end of the section.

We can now prove the lifting property $L(i)$. where we are given a diagram in tow-S

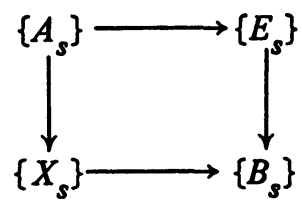

in which $\left\{A_{s}\right\} \rightarrow\left\{X_{s}\right\}$ is a cofibration and $\left\{E_{s}\right\} \rightarrow\left\{B_{s}\right\}$ is a trivial level-fibration. As before, we can assume that in fact we have a level diagram

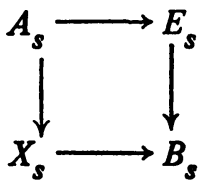

in which each $A_{s} \rightarrow X_{s}$ is a cofibration and $E_{s} \rightarrow B_{s}$ satisfies Lemma 8.2. To simplify notation, we write $X_{s}^{(r)}$ for $A_{s} \cup X_{s}^{r}$. For this proof let $N(s)$ be an integer greater than $N(s-1)+2$, such that $E_{s} \rightarrow B_{s}$ is a co- $N(s)$-equivalence; $N(-1)=0$. Let $p(s)=s+N(s)+1$.

First, for $s=0$, we construct $X_{p(0)} \rightarrow E_{0}$ in stages $X_{p(0)}^{(k)} \rightarrow E_{p(0)-k-1}$ for $k=0,1, \cdots, p(0)-1=N(0)$. Consider the following diagram:

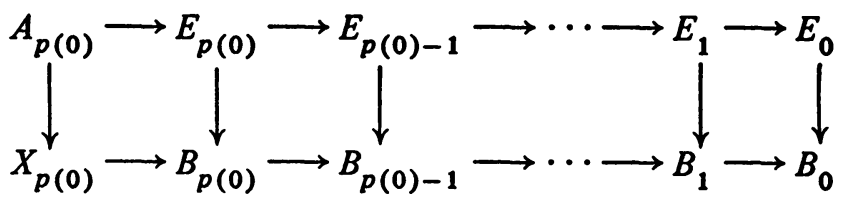

By Lemmas 8.1 and 8.2 (i) the desired liftings $X_{p(0)}^{(k)} \rightarrow E_{p(0)-k-1}$ exist. Further, by Lemma 7.2 and the choice of $N(0)$, we can extend $X_{p(0)}^{(N(0))} \rightarrow$ $E_{0}$ to $X_{p(0)} \rightarrow E_{0}$.

Now assume by induction on $s$ that we have compatible liftings $X_{p(s-1)}^{(k)} \rightarrow E_{p(s-1)-k-1}$ for $k=0,1, \cdots, p(s-1)-s=N(s-1)$ and $X_{p(s-1)} \rightarrow E_{s-1}$. We wish to construct maps $X_{p(s)}^{(k)} \rightarrow E_{p(s)-k-1}$ for 
$k=0,1, \cdots, p(s)-s-1=N(s)$ and $X_{p(s)} \rightarrow E_{s}$, compatible with each other and with the skeleta liftings for $s-1$. Define $E_{p(s)-r}^{\prime}$ by the following rules:

$$
\begin{aligned}
& E_{p(s)-r}^{\prime}=B_{p(s)-r} \times_{B_{p(s-1)-r-1}} E_{p(s-1)-r-1}, \quad 0 \leqslant r<p(s-1)-s, \\
& E_{p(s)-r}^{\prime}=B_{p(s)-r} \times_{B_{s-1}} E_{s-1}, \quad p(s-1)-s \leqslant r \leqslant p(s)-s .
\end{aligned}
$$

Then there are natural liftings $X_{p(s)}^{(r)} \rightarrow E_{p(s)-r}^{\prime}$ for $0 \leqslant r<p(s-1)-s$ and $X_{p(s)} \rightarrow E_{p(s)-r}^{\prime}$ for $p(s-1)-s \leqslant r \leqslant p(s)-s$, since $X_{p(s)}^{(r)}$ maps to $E_{p(s-1)-r-1}$ through $X_{p(s-1)}^{(r)}$, and $X_{p(s)}$ maps to $E_{s-1}$ through $X_{p(s-1)}$. We wish to construct the dotted arrow in the following diagram

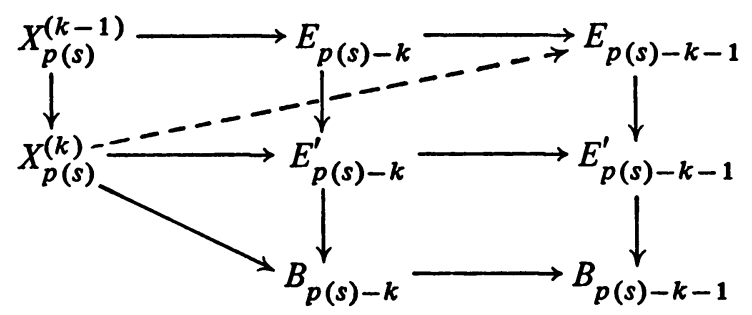

where we are assuming by induction on $k$ that $X_{p(s)}^{(k-1)} \rightarrow E_{p(s)-k}$ has already been constructed (there being nothing to prove for $k=-1$ ). There are two cases. If $0 \leqslant k<p(s-1)-s$, we have the situation of Lemma 8.2 (ii) and the lifting exists by Lemma 8.1. Suppose therefore that $p(s-1)-s \leqslant k<$ $p(s)-s$. In order to apply Lemma 8.1 we want, for every choice of basepoint in $E_{p(s)-k}$, that $\pi_{k-1}\left(G_{p(s)-k}, *\right) \rightarrow \pi_{k-1}\left(G_{p(s)-k-1}, *\right)$ be zero, where $G_{i}$ is the fiber of $E_{i} \rightarrow E_{i}^{\prime}$. First note that the fiber $H$ of $E_{p(s)-k-1}^{\prime} \rightarrow$ $B_{p(s)-k-1}$ is the same as the fiber of $E_{s-1} \rightarrow B_{s-1}$ and in particular $\pi_{k}(H, *)=0$ since $k \geqslant N(s-1)$. Now since $\pi_{k-1}\left(F_{p(s)-k}, *\right) \rightarrow$ $\pi_{k-1}\left(F_{p(s)-k-1}, *\right)$ is zero by Lemma 8.2, it suffices to show that $\pi_{\dot{k}-1}\left(G_{p(s)-k-1}, *\right) \rightarrow \pi_{k-1}\left(F_{p(s)-k-1}, *\right)$ is monomorphic. For this we apply the Five Lemma to the following diagram (* and subscripts $p(s)-k-1$ are suppressed):

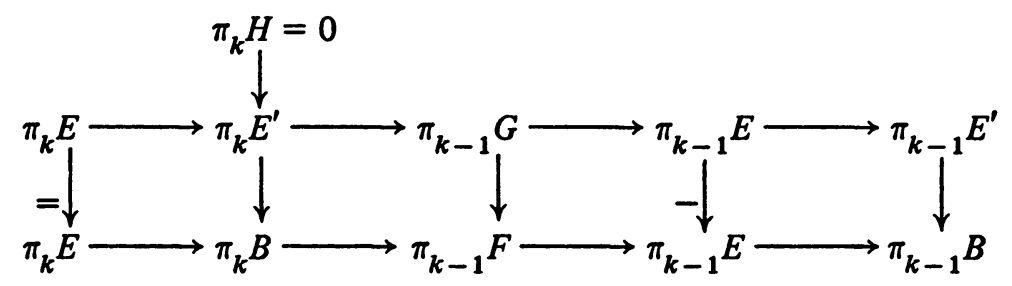

Having constructed the skeleton lifting $X_{p(s)}^{(p(s)-s-1)} \rightarrow E_{s}$, the dotted arrow finally exists in the following diagram by Lemma 7.2 and the choice of $p(s)$ : 


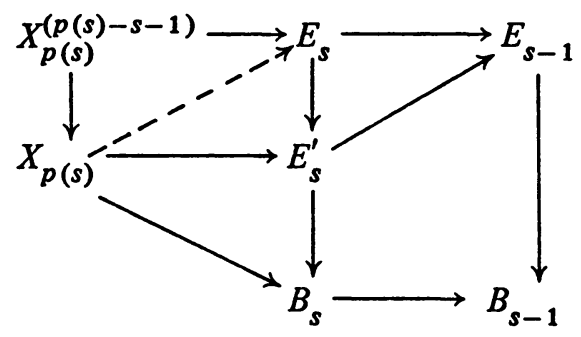

Proof OF 8.2. We start with any level representative $\left\{E_{s} \rightarrow B_{s}\right\}$ of the trivial level-fibration which satisfies Definition 4.5. By the definition of weak equivalence (4.3) and level-fibration (4.5) we can, by taking a subtower, assume that for each $s \geqslant 0$ the dotted arrow exists in the following diagram

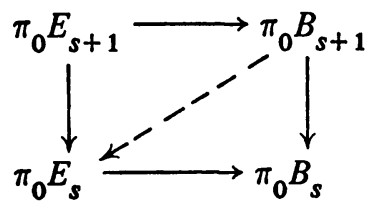

and for each $n \geqslant 1$ and each choice of basepoint in $E_{s+1}$ the dotted arrow exists in the following diagram: •

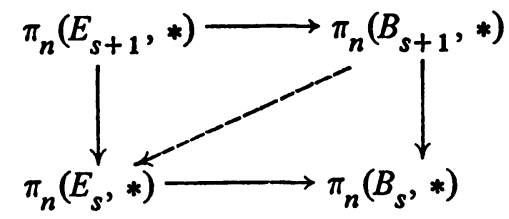

A diagram chase in the long exact sequence of homotopy groups of the fibrations $E_{s} \rightarrow B_{s}$ shows that $\pi_{n}\left(F_{s+2}, *\right) \rightarrow \pi_{n}\left(F_{s}, *\right)$ is the zero map for each $s \geqslant 0, n \geqslant 0$, and basepoint in $E_{s+2}$. The subtower $\left\{E_{2 s} \rightarrow B_{2 s}\right\}$ then satisfies condition (i). A lengthy diagram chase now shows that condition (ii) will be satisfied as well if one takes the subtower $\left\{E_{4 s} \rightarrow B_{4 s}\right\}$. By the comment following Definition 4.5, the final subtower still satisfies Definition 4.5.

\section{BIBLIOGRAPHY}

1. M. Artin and B. Mazur, Étale homotopy, Lecture Notes in Math., no. 100, Springer-Verlag, Berlin and New York, 1969. MR 39 \#6883.

2. A. K. Bousfield and D. M. Kan, Homotopy limits, completions, and localizations, Lecture Notes in Math., vol. 304, Springer-Verlag, Berlin and New York, 1972.

3. E. Dror, Pro-nilpotent representation of homology types, Proc. Amer. Math. Soc. 38 (1973), 657-660.

4. J. P. May, Simplicial objects in algebraic topology, Van Nostrand Math. Studies, no. 11, Van Nostrand, Princeton, N. J., 1967. MR 36 \#5942.

5. D. G. Quillen, Homotopical algebra, Lecture Notes in Math., no. 43, SpringerVerlag, Berlin and New York, 1967. MR 36 \$6480. 
6. D. G. Quillen, Rational homotopy theory, Ann. of Math. (2) 90 (1969), 205295. MR 41 \#2678.

7. E. H. Spanier, Algebraic topology, McGraw-Hill, New York, 1966. MR 35 \#1007.

DEPARTMENT OF MATHEMATICS, MASSACHUSETTS INSTITUTE OF TECHNOLOGY, CAMBRIDGE, MASSACHUSETTS 02139

Current address: Department of Mathematics, Oakland University, Rochester, Michigan 48063 